\title{
A Verified Algebraic Representation of Cairo Program Execution
}

\author{
Jeremy Avigad \\ avigad@cmu.edu \\ Carnegie Mellon University \\ USA
}

\author{
Lior Goldberg \\ lior@starkware.co \\ StarkWare Industries Ltd. \\ Israel
}

\author{
David Levit \\ david@starkware.co \\ StarkWare Industries Ltd. \\ Israel
}

\author{
Yoav Seginer \\ yseginer@gmail.com \\ Netherlands
}

\author{
Alon Titelman \\ alon@starkware.co \\ StarkWare Industries Ltd. \\ Israel
}

\begin{abstract}
Cryptographic interactive proof systems provide an efficient and scalable means of verifying the results of computation on blockchain. A prover constructs a proof, off-chain, that the execution of a program on a given input terminates with a certain result. The prover then publishes a certificate that can be verified efficiently and reliably modulo commonly accepted cryptographic assumptions. The method relies on an algebraic encoding of execution traces of programs. Here we report on a verification of the correctness of such an encoding of the Cairo model of computation with respect to the STARK interactive proof system, using the Lean 3 proof assistant.
\end{abstract}

CCS Concepts: • General and reference $\rightarrow$ Verification; - Theory of computation $\rightarrow$ Logic and verification; • Software and its engineering $\rightarrow$ Semantics.

Keywords: interactive proof systems, formal verification, smart contracts

\section{ACM Reference Format:}

Jeremy Avigad, Lior Goldberg, David Levit, Yoav Seginer, and Alon Titelman. 2022. A Verified Algebraic Representation of Cairo Program Execution. In Proceedings of the 11th ACM SIGPLAN International Conference on Certified Programs and Proofs (CPP '22), Fanuary 17-18, 2022, Philadelphia, PA, USA. ACM, New York, NY, USA, 13 pages. https://doi.org/10.1145/3497775.3503675

\section{Introduction}

The execution of smart contracts [30] on blockchain makes it possible to carry out transactions such as cryptocurrency

This work is licensed under a Creative Commons Attribution 4.0 International License.

CPP '22, January 17-18, 2022, Philadelphia, PA, USA

(C) 2022 Copyright held by the owner/author(s).

ACM ISBN 978-1-4503-9182-5/22/01.

https://doi.org/10.1145/3497775.3503675 exchanges and auctions in the absence of institutional oversight. But the technology faces a scaling problem: distributed blockchain protocols require anyone verifying the integrity of the blockchain to carry out the computation associated with the contract, constituting a draw on resources.

Seminal work of Babai, Fortnow, and Lund [8] showed that interactive proof protocols, introduced to complexity theory in the mid-1980s [7, 20], allow for efficient verification of computational claims. They provide protocols by which a computationally powerful prover can convince a computationally limited verifier that the execution of a program on a given input yields a certain result, without requiring the verifier to execute the program itself. The original protocols rely on a source of randomness to convince the verifier that the claim holds with high probability. Contemporary variants replace the use of randomness by the use of cryptographic methods and assumptions.

Here we focus on the use of one particular cryptographic protocol, the STARK protocol [10], to verify claims about execution in one particular Turing-complete model of computation, the Cairo machine [19]. The Cairo model of computation is a machine with a small instruction set and the ability to read from a bank of memory consisting of values in a finite field. Roughly speaking, the STARK protocol allows a prover to efficiently convince a verifier that the prover possesses a table of values from a large finite field, such that tuples from the table satisfy a family of polynomials that is shared by both the prover and verifier. In our case, this table of values encodes the trace of an execution of the Cairo machine.

To employ the STARK protocol, claims about the execution of a Cairo program on a given partial assignment to memory are encoded using an algebraic intermediate representation (AIR). Specifically, the claim that a program terminates successfully is expressed as a claim about the existence of a solution to a family of polynomials, which is exactly the type of claim that can be verified by the STARK protocol.

When the execution of a smart contract can have substantial financial repercussions, it is important to have prior confidence that the outcome will be as intended. In that 
respect, there are at least three aspects of the process just described that are open to formal verification. First, one may wish to verify the claimed properties of STARKs, that is, the claim that the protocol establishes the existence of the data satisfying the polynomial constraints with high probability, under the relevant cryptographic assumptions. Second, one may wish to verify the algebraic representation of program execution, that is, the claim that the existence of data satisfying the AIR implies the existence of an execution trace of the corresponding program. Finally, one may wish to verify claims that particular Cairo programs meet their specifications, for example, that the successful execution of a program correctly determines the outcome of an exchange.

Although the first task would constitute an interesting formalization project, we take the other two tasks to be more pressing. Cryptographic protocols have been well-studied and the papers on STARKs and related protocols have appeared in peer-reviewed journals, so it seems reasonable to treat the protocol as a black box for now. Work on the third task is in progress, but we will not report on that here. This paper reports on the successful completion of the second task, a fully verified proof that data satisfying the AIR implies the corresponding computational claim. The AIR verified by the proof is directly generated from the Cairo implementation, allowing for continuous verification of the AIR used in production.

When formal verification and computational complexity come together, the phrase "interactive proof system" is used in two unrelated ways. In formal verification, it refers to a computational proof assistant, whereas in complexity theory and cryptography, it refers to the kind of interactive proof protocols described above. The terminology is standard in both communities, and it is generally not hard to resolve the ambiguity in context.

Our formalization follows the informal proofs-some presented in detail, others sketched-in what we will refer to as the Cairo whitepaper [19]. The most up-to-date version of the formalization, as well as instructions for compiling or browsing it in Lean, can be found at https://github.com/starkwarelibs/formal-proofs. In the PDF version of this paper, all . lean file references are hyperlinked to the files in this repository.

\section{The Formalization Platform}

Our formalization is carried out in the Lean 3 proof assistant [16] with its associated library, mathlib [26]. Lean's axiomatic foundation is a version of dependent type theory with inductive types and a type of propositions. The core logic is constructive, but we (and mathlib) make free use of classical logic.

Our formalization does not depend heavily on the specifics of Lean and mathlib, however. We use basic facts from the library about natural numbers, integers, finite indexing types, bitvectors, and finite fields. Perhaps the most mathematically involved fact we need is that a degree $n$ polynomial over a field has at most $n$ roots. We also make moderate use of automation. Lean's simplifier, which does conditional term rewriting with a battery of tagged and/or explicitly enumerated rewrite rules, has been helpful, especially for the parts of the formalization described in Section 8. We use a tactic, norm-num, that carries out verified numeric calculations.

\section{The Cairo CPU}

The successful run of a Cairo program on a given input is meant to convince a skeptical verifier of some claim about those inputs. An unusual feature of the model of computation is that memory is read only. When executing a Cairo program and preparing the data needed for the interactive proof protocol, it is the prover's obligation to arrange data in memory so that the program executes successfully.

The computation model is based on a CPU with three registers, a program counter (pc), an allocation pointer (ap), and a frame pointer (fp), each of which points to locations in the read-only memory. The program counter contains the memory address of the instruction that is about to be executed. By convention, the allocation pointer points to a yet-unused memory cell, and it usually only increases as a program executes. The frame pointer is used to point to a function's local memory. When a function is called, the frame pointer is set equal to the allocation pointer, and when the function returns, the frame pointer is restored to the value it had before the previous call, that is, the address of the calling function's local memory.

In the Cairo model, register values, as well as values in memory, are elements of a finite field, $F$. As a result, there is no order on the elements; a program can test equality of elements but cannot compare them to determine which is greater. Using a field means that we can add, subtract, multiply, and divide. Program instructions are 63 bits long, and so can be represented by an integer less than $2^{63}$. The semantics assumes (and the algebraic data presented to the verifier guarantees) that the characteristic of the underlying field is greater than $2^{63}$, which means that instructions have a unique representation in the field. But algebraic constraints are needed to guarantee that a given field element represents an instruction in such a way, as well as to reason about the instruction's components. We will explain how this works in Section 7.

The fact that memory is read only means that we do not need to verify claims about memory management. Another consequence of the intended use is that we do not need to prove that a program terminates; the algebraic data verified by the interactive proof system guarantees that the program has run for a certain number of steps, after which the program counter reached a certain value. That is not to say that it would not be helpful to also have general guarantees that high-level Cairo programs terminate and manage memory 
appropriately; such completeness claims guarantee that the prover can publish the desired proofs. At present, extensive testing and code review is used for that purpose, and establishing soundness, i.e. the fact that the proofs do what they are supposed to, is the more pressing concern.

There are a few basic types of Cairo machine instructions:

- assert statements, which assert equality between two values

- conditional and unconditional jumps, the former based on a test for zero

- call and return

- an instruction to advance the allocation pointer.

Arguments can refer to the contents of memory locations using offsets from the frame pointer or allocation pointer. They also support addition and multiplication. Each instruction contains three bitvectors of length 16, which can provide, for example, memory offsets for the operands and result of an assert. Each instruction also contains 15 1-bit flags, which determine things like the type of instruction, the nature of the operands, and whether the allocation pointer should be augmented after the instruction.

An instruction is formally represented in Lean as follows:

$$
\begin{aligned}
& \text { structure instruction := } \\
& \text { (off_dst : bitvec 16) } \\
& \text { (off_op0 : bitvec 16) } \\
& \text { (off_op1 : bitvec 16) } \\
& \text { (flags : bitvec 15) }
\end{aligned}
$$

For example, in the Cairo assembly language, we might write an instruction as follows:

$$
[a p+10]=[\mathrm{fp}] *[\mathrm{fp}-1] \text {. }
$$

This asserts that the value in memory at location ap +10 is the product of the values at locations $f p$ and $f p-1$, where ap and $f p$ denote the allocation pointer and frame pointer, respectively. This instruction is encoded in the structure above by setting the flags to specify that the operation is an assert, that the relevant operation is multiplication, that the operands are addressed by offset from $\mathrm{fp}$, and that the result is addressed by offset from $f p$. The values of off_dst, off_op 0 , and off_op1 represent 10, 0, and -1, respectively. The instruction is ultimately converted to a number, which, in turn, is stored in memory as an element of F. Some instructions also require an immediate value, which is stored in the next element of memory.

The register state is represented formally as follows:

$$
\begin{aligned}
& \text { structure register_state }(F: \text { Type* }):= \\
& (p c: F)(a p: F)(f p: F)
\end{aligned}
$$

A single state of the virtual Cairo machine is given by the contents of memory, which we model as a function from $F$ to $F$, and the current register state. Given such a state, the Cairo whitepaper [19] specifies the potential successor states. The successful execution of a well-formed instruction leads to a unique successor state. But there may not be a successor state, for example, when an assertion fails because in the given state the asserted equality doesn't hold. And ill-formed instructions result in undefined behavior, giving rise to the possibility of multiple successor states. We therefore use a next-state relation to model the step semantics. The file cpu. lean, less than 200 lines, is a straightforward formalization of the whitepaper description, culminating in the following definitions:

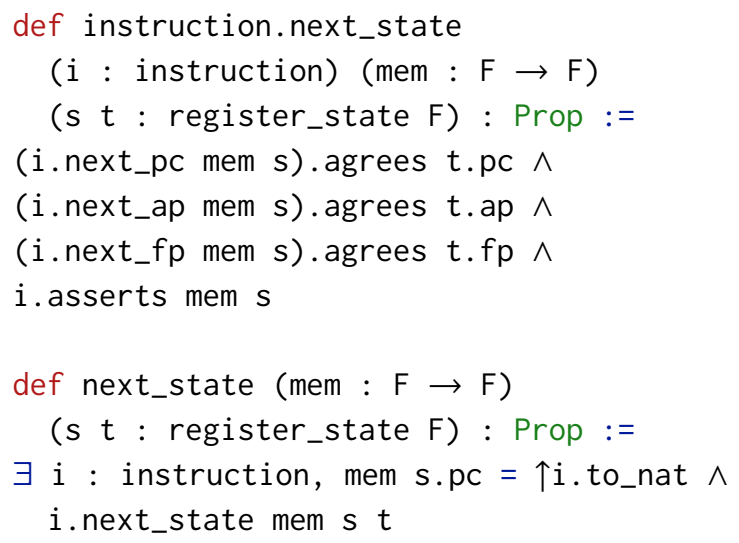

The first definition says that $t$ is a successor state to $s$ assuming $i$ is the current instruction. It relies on auxiliary definitions that specify the next program counter, the next allocation pointer, the next frame pointer, and any assertions associated with the instruction. Notation such as i .next_pc relies on a nice bit of Lean syntax known as "anonymous projections": given $i$ of type instruction and a definition instruction.next_pc in the environment, Lean interprets i.next_pc mem s as instruction.next_pc i mem s. (In this example, $i$ is inserted as the first argument of type instruction.) Each of the functions next_pc, next_ap, and next_fp returns an element of an option type, equal to none if the instruction results in undefined behavior. If $x$ is an element of an option type, the relation $x$. agrees a says that if $x$ is of the form some $b$, then $a$ is equal to $b$.

The second definition, the next_state relation, simply asserts that the memory at the program counter contains the cast of an instruction to the field and that the next state agrees with the one corresponding to that instruction.

Other definitions specify the way that the value of the next state is determined based on the instruction flags and offset values. For example, the next value of the frame pointer depends on whether the current instruction is a call, a return, an assert, or none of the above. The specification has the following shape, where the auxiliary function instruction.dst mem $s$ determines the appropriate value for restoring the frame pointer on the return from a function call.

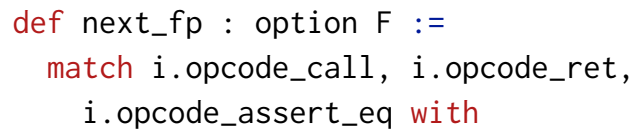




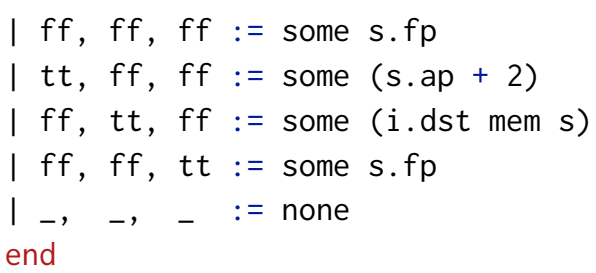

The function is a case distinction on the settings of the corresponding flags of the instruction. After a call instruction, for example, the frame pointer is set to the current allocation pointer plus 2. After a return instruction, the next value of the frame pointer is computed using the function instruction.dst. The other instructions do not change the frame pointer.

The Cairo toolchain allows programmers to write Cairo programs in an assembly language that is close to the machine code, with instructions like these:

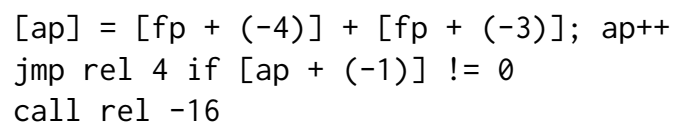

The first instruction says that the value of the memory location referenced by the allocation pointer is equal to the sum of the values at two locations referenced using the frame pointer. The ap++ suffix specifies that the instruction also increments the allocation pointer. The next instruction is a conditional relative jump, and the third executes a call to the function at the current program counter minus 16 .

The Cairo toolchain also allows programmers to write programs in a higher-level language with function definitions, data structures, conditional blocks, and recursive function calls. These are compiled to assembly instructions, which are encoded as machine instructions and ultimately as field elements that are shared with the verifier.

\section{The AIR}

In the intended scenario, the prover and the verifier agree on a Cairo program of interest and the input to that Cairo program. The prover wants to convince the verifier that executing the program on the inputs yields a certain output. The prover does this by publishing a proof, a suitable certificate, at which point the verifier executes an algorithm that uses the certificate to ascertain the correctness of the claim. The catch is that, in the intended usage, the certificate will be published on blockchain and the verifier will be executed as part of a smart contract, so we want the certificate to be small and the verification to be substantially more efficient than executing the Cairo program itself.

Whereas the original interactive proof systems made use of randomness to achieve such goals, blockchain protocols rely on the use of cryptographic hash functions instead of coin flips. The effectiveness of the proof relies on the assumption that a dishonest but computationally-bounded prover does not have a substantially better than random chance of gaming the use of a hash.

Our method relies on the STARK protocol, which works roughly as follows. Fix a large prime number $p$, and let $F_{p}$ be the finite field of integers modulo $p$. The STARK protocol allows the prover to convince the verifier that the prover is in possession of a two-dimensional table of field elements of $F_{p}$ that satisfies a constraint system that the prover and verifier agree on. The constraint system is given as a list of polynomials $P_{1}\left(\vec{x}_{1}\right), \ldots, P_{s}\left(\vec{x}_{s}\right)$ over $F_{p}$, and a corresponding list of domains $D_{1}, \ldots, D_{s}$, which are periodic subsets of the row indices. The arguments of the polynomials can be taken from multiple rows and multiple columns of the table. We say that the constraint system is satisfied by a certain table with $N$ rows if for every $j=1, \ldots, s$ and every $r \in D_{j}$, we have $P_{j}\left(\vec{x}_{j}^{(r)}\right)=0$, where $\vec{x}_{j}^{(r)}$ is $\vec{x}_{j}$ shifted by $r$ rows. For example, a polynomial $P(\vec{x})$ might involve variables in two consecutive rows, and the corresponding set $D$ might specify that the constraint holds at every row in the table, or that it holds at every other row, or that it holds at every fourth row. In our application, the polynomials are fixed modulo some field parameters that the prover and verifier share. The verification algorithm runs in time polynomial in the logarithm of $N$ and the size of the parameters that the prover and verifier share, and it provides high assurance that the result is correct.

To meet our goals, it therefore suffices to design a sequence of polynomials $P_{1}\left(\vec{x}_{1}\right), \ldots, P_{s}\left(\vec{x}_{s}\right)$ such that, for parameters corresponding to the Cairo program, input data, and output in question, and for a suitable $N$, the existence of a table of data meeting the criteria described in the previous paragraph implies the existence of an execution sequence of the given Cairo program on the given input data, yielding the given output.

For the purposes of this paper, the details of the STARK protocol and the precise complexity and probabilistic claims can be treated as a black box. Our goal here is to describe the polynomials $P_{1}, \ldots, P_{s}$ and to describe a formal proof that the existence of the table of data implies the existence of the desired execution sequence. Assuming the verifier trusts the STARK protocol, the successful run of the verification algorithm yields a strong guarantee of the existence of a table of data satisfying the constraints, and our formal proof turns this into a strong guarantee that the Cairo program executes as claimed.

The constraints $P_{1}, \ldots, P_{s}$, as well as the relevant parameters, are listed in the file constraints_autogen. lean. As the name suggests, this file is automatically generated. In fact, it is generated by the same code that produces the code for the verifier. This, together with the specification of the CPU semantics in cpu. lean, are the only two files that the statement of our final theorem depends on, other than general basic facts about data structures, fields, and so on in the 
library. The desired conclusion, namely, the existence of an execution trace consistent with the given partial assignment to memory, relies on the fact that the STARK certificate that is verified on the blockchain is correct with respect to these polynomial constraints and parameters. A skeptic can inspect the smart contract that performs the verification to ensure that this is the case.

The fact that the Cairo program, the input, and the output are all stored in memory allows us to simplify the description of the verification task. It suffices for the prover and verifier to agree on a partial assignment of values to the memory that includes that data and the initial state of the CPU. In fact, we share that information, the length of the execution trace, and the final value of the program counter and allocation pointer; the polynomial constraints ensure that the initial value of the frame pointer is equal to the initial value of the allocation pointer. This data is represented as follows:

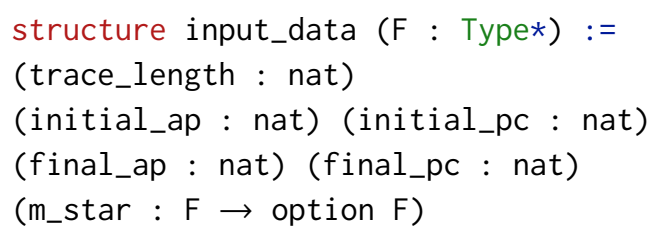

The name m_star corresponds to the fact that the partial assignment is written as $\mathrm{m}^{*}$ in the whitepaper, and the return type, option $F$, means that the function can either return an element of the type some $a$, where $a$ is an element of $F$, or none.

The publicly shared parameters also include additional data, including information about range checked elements and the interaction elements; these are explained in Sections 9-11 below.

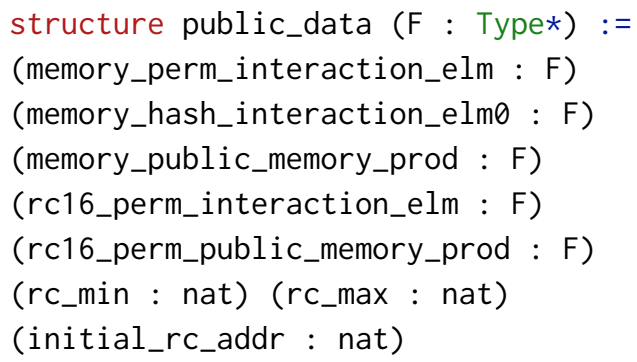

The correctness proof assumes that this data satisfies certain assumptions that can be easily confirmed by the verifier.

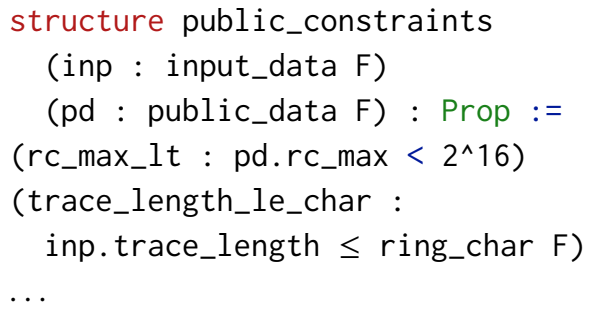

Suppose the Cairo program terminates after $T$ steps. The execution trace then consists of $T+1$ register states, including the start state and end state, and depends on $T$-many instructions in the memory along the way. Without loss of generality, we can assume that $T+1$ is a power of two, since, by convention, Cairo programs "terminate" by entering an infinite loop. The data in the Algebraic Intermediate Representation (AIR) consists of a list of $16(T+1)$-many tuples, each consisting of 25 columns. These are used to encode the execution trace, the instructions, and the contents of memory, in ways that we will describe below. The parameter trace_length in the structure input_data indicated above is actually $16(T+1)$, corresponding to the parameter $N$ described earlier in this section. We recover $T$ by dividing by 16 and subtracting one. We often refer to the elements of the AIR as trace cells, since they encode the states of the execution trace, as well as memory accesses along the way and auxiliary data.

We can now state our main result: our formal proof shows that satisfaction of the polynomial constraints guarantees that the encodings have the claimed meaning with respect to the formal semantics. In other words, the existence of a table of data satisfying the contraints implies the existence of an execution trace that is consistent with the agreed-upon partial assignment to memory. For the protocol, it is important that the prover commit to the first 23 columns of data before "random" interaction elements are computed based on a cryptographic hash of the first columns; the remaining 2 columns then depend on the result of these interaction elements. From the published data the verifier can ensure that this is, in fact, the case, and in the next section we will see how they are incorporated into the correctness proof.

The Cairo whitepaper describes the relevant polynomials and encoding of data without specifying exactly how the data is laid out in the $16(T+1) \times 25$ array. This makes the constraints easier to read and understand. Our initial formalization followed the whitepaper; the file constraints. lean formalizes those constraints, and the file correctness. lean proves the correctness theorem in those terms. We then incorporated a file glue. lean that mediates between the two representations, resulting in the end-to-end correctness proof in final_correctness. lean.

\section{The Correctness Theorem}

The statement of the final correctness theorem depends only on the specification of the Cairo execution semantics in cpu. lean and the autogenerated file of constraints, constraints_autogen.lean. The full statement of the theorem is presented in Figure 1.

In the statement of the theorem, $F$ is assumed to be a finite field of characteristic at least $2^{63}$. We are given the shared input data, inp : input_data $F$, and the remaining public data, pd : public_data F, assumed to satisfy the 
required constraints. We are also given c: columns $F$, a structure that consists of 23 columns named c. column 0 to c. column22.

Based on this data, we assert the existence of three "bad" subsets bad1, bad2, bad3 of the field $F$. Letting $T^{\prime}$ be the shared input parameter inp.trace_length, these are asserted to have cardinality at most $\left(T^{\prime} / 2\right)^{2}, T^{\prime} / 2$, and $T^{\prime}$, respectively. The idea is that for the execution sequences that arise in practice, these values will be substantially smaller than the cardinality of $F$, so that a randomly chosen element of the field is unlikely to land in the bad sets. As we explain in Section 9, if the prover has encoded the relevant data honestly, these sets are all empty, but if the prover is dishonest, the prover will be caught in the lie unless the interaction elements happen to return values in these small sets. Once these bad sets are determined, the prover commits to the final two columns, ci : columns_inter F.

The theorem then says that if the columns $c$ and ci satisfy all the polynomial constraints, and assuming that the generated interaction elements are not in the small bad sets, then there exists an execution trace of the Cairo program meeting the agreed-upon specification.

In greater detail, we assume that the data c, ci, inp, and pd satisfies the autogenerated constraints

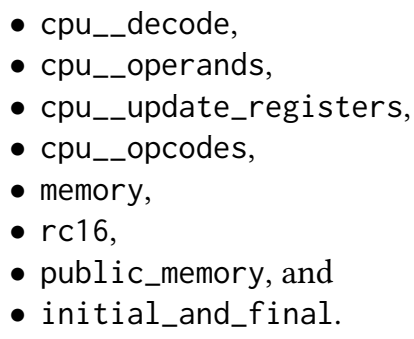

We will describe the contents of these below. We also assume that the interaction elements have landed outside the small bad sets that depend only on the first 23 columns. Under the cryptographic assumptions, this occurs with high probability. Let $T$ be $\left(T^{\prime}-1\right) / 16$. The theorem then asserts that there exists an assignment to the memory that extends the agreedupon partial assignment and an execution trace of the Cairo program, for that memory assignment, that starts at the start state, runs for $T$ steps, and ends at the end state. In other words, the first and last state of the execution trace are as claimed, and each successive state follows the previous one according to the machine semantics specified in cpu. lean.

What needs to be trusted? The polynomials that are found in constraints_autogen. lean should match the polynomials used by the verifier. Since the definition of a field, the definition of integers, finite sequences, and so on come into the definition of the semantics and the statement of correctness, one needs to assume that they have been formalized correctly, so that, for example, a statement about a finite field really is a statement about a finite field. Since the formalization says something about the CPU semantics,

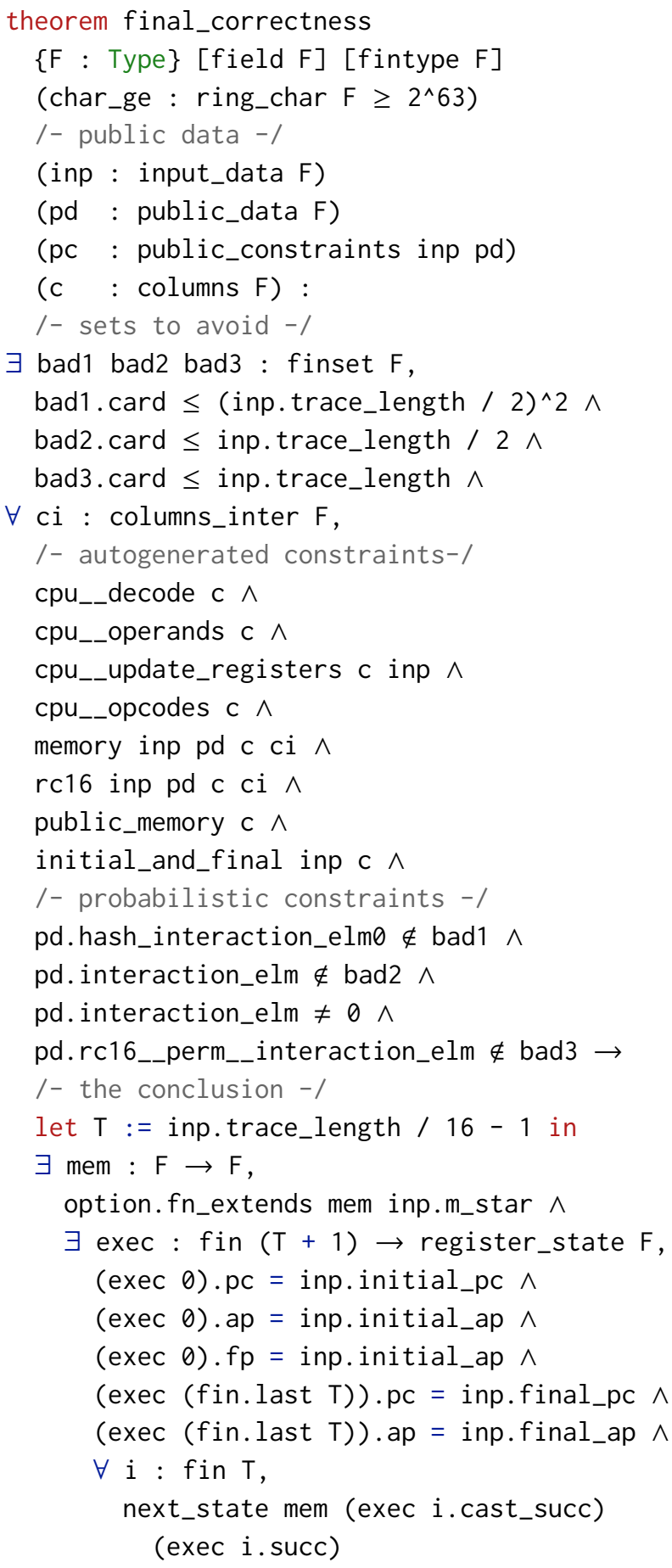

Figure 1. The final correctness theorem.

we have to accept that the formalization of that semantics matches our informal understanding. However, if we are later able to prove that programs meet high level specifications with respect to the semantics (which we intend to 
do, as described in Section 1), at that point only the higherlevel specification matters; the execution semantics becomes only a stepping-stone to the final result. Of course, we have to trust the soundness of the axiomatic foundation and its implementation in Lean. Finally, as described in Section 1, an implementation of the method also requires trusting the STARK protocol and the means used to verify the STARK certificates.

Once we trust the formalization, we do not need to worry about why or how the polynomial constraints guarantee the existence of the final execution trace. The formal proof establishes that it does. From the point of view of the design of the system, however, and from the point of view of verification, this is the most interesting part. The details are spelled out in the Cairo whitepaper [19]. In the sections that follow we sketch the proof and provide some indications as to how it is formalized.

\section{Summary of the Constraints}

Altogether, the Cairo algebraic intermediate representation of an execution trace is encoded by the following constraints:

- constraints for specifying and decoding instructions;

- constraints that specify the next-step relation, and, in particular, specify the operands for an assert statement and the values of the program counter, frame pointer, and allocation pointer, all in terms of the current instruction and values in memory;

- constraints that show that the prover has an assignment of values to memory that is consistent with program execution and extends the partial assignment that the prover and verifier have agreed upon; and

- constraints that guarantee that memory addresses and instruction components are integers in the required range.

We describe each of these categories, in turn, in the sections that follow.

The full list of constraints is found in Section 9.10 of the Cairo whitepaper [19]. They are stated as local hypotheses in the files throughout the formalization, and they are gathered into structures in the file constraints. lean. That file provides the parameters and hypotheses that are used in the statement of the correctness theorem in correctness. lean. The file constraints_autogen. lean contains a description of the constraints that is generated automatically from the Cairo source code, and so reflects the data that is used to generate the STARK certificate. It is these latter constraints that the verifier can check using the STARK protocol. We therefore use the file glue. lean to instantiate the structures and hypotheses in constraints. lean, and the final correctness theorem in Figure 1 is stated in terms of the autogenerated constraints. This means that the verifier does not have to trust any aspect of the implementation: the STARK certificate ensures that the constraints are met, and the formal theorem shows that this implies the existence of the claimed execution trace.

\section{Decoding Instructions}

In Section 8, we will discuss polynomial constraints that, given an instruction $i$, the contents of memory $m$, and a register state $s$, specify that another register state $t$ follows $s$ according to $i$. For that purpose, however, we need to reason about the components of $i$. We also need to ensure that the field element $i$ is, in fact, a valid instruction, which is to say, it is the result of casting an integer in $\left[0,2^{63}\right)$ to the finite field. Note that we can always use auxiliary elements of the AIR when writing constraints. These are existentially quantified by the STARK protocol.

Remember that an instruction contains three 16-bit num-

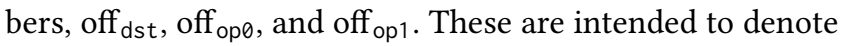
integer offsets in $\left[-2^{15}, 2^{15}\right)$, but in the AIR we store the corresponding elements $\widetilde{o f f}_{*}=$ off $_{*}+2^{15}$ (where $*$ is one of op0, op1, or dst). We also need to reason about the flags $f_{0}, \ldots, f_{14}$. Rather than using trace elements of the AIR for these, we define $\tilde{f}_{i}=\sum_{j=i}^{14} 2^{j-i} \cdot f_{j}$ and store $\tilde{f}_{0}, \ldots, \tilde{f}_{15}$. Note that this means that $\tilde{f}_{0}=\sum_{j<15} 2^{j} \cdot f_{j}$ is the integer in $\left[0,2^{15}\right.$ ) with bits $f_{0}, \ldots, f_{14}$, and that $\tilde{f}_{15}=0$. We can recover the bits $f_{i}$ with the identity $f_{i}=\tilde{f}_{i}-2 \tilde{f}_{i+1}$.

In Section 10 we explain how the AIR can constrain the values $\widetilde{\text { off }}_{*}$ to be integers in the range $\left[0,2^{16}\right)$. Setting that aside, the AIR should therefore assert that elements $\tilde{f}_{i}$ encode a sequence of $0 \mathrm{~s}$ and $1 \mathrm{~s}$ via the identity above, and that the value representing the instruction is equal to off $\widetilde{d s t}+2^{16}$. $\widetilde{\text { off }}_{\mathrm{op} \theta}+2^{32} \cdot \widetilde{\mathrm{off}}_{\mathrm{op} 1}+2^{48} \cdot \tilde{f}_{0}$. These constraints are written in Lean as follows:

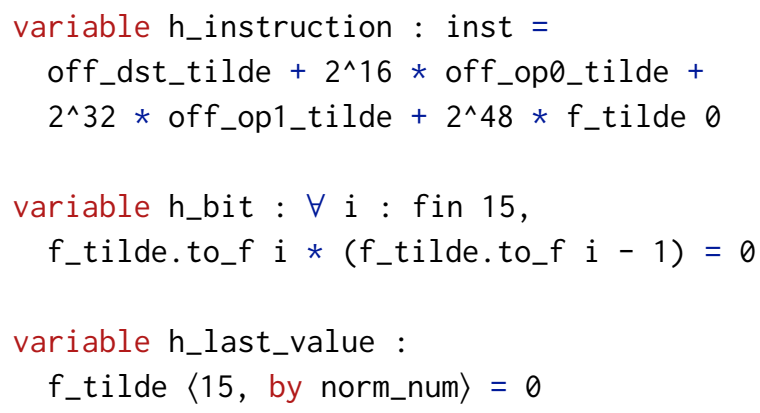

To make sense of this, note that in Lean, a hypothesis is encoded as a variable whose type is the statement in question. The type fin 15 consists of the numbers from 0 to 14 , which are used to index the bits. In contrast, the variables $\tilde{f}_{i}$ are indexed by elements of fin 16, and the Lean idiom $\langle 15$, by norm_num $\rangle$ is used to denote the last element of this type and automatically fill in the proof that 15 is less than 16 . The to_f function in the expression $f_{\text {_tilde.to_f }} \mathrm{i}$ computes the value of $f_{i}$ from the tuple $\tilde{f}$ via the identity above.

Theorem 1 of the whitepaper asserts that with these constraints (and the ones that ensure that the values $\widetilde{\text { off }}_{*}$ are 
integers in the right range) there is a unique instruction such that the field element inst encodes that instruction. In our formalization, we generally need to refer to the instruction encoded by inst on the assumption that these constraints are met. Therefore, our formalization takes the following form First, we define a function

$$
\text { def the_instruction : instruction : }=\ldots
$$

This specifies the instruction from the data $\widetilde{\text { off }}_{*}$ and $\tilde{f}$. (In Lean, when variables and hypotheses are declared in a file, definitions that use them can leave the dependence implicit. The dependencies are part of the definition, however, and are displayed when the user asks Lean to show an expression or its type.) Then, we prove that, assuming the constraints are met, the field element is equal to the cast (to the field) of the natural number encoding of the instruction, and the trace elements $\widetilde{o f f}_{*}$ are the results of casting the components of the instruction to the field. For example:

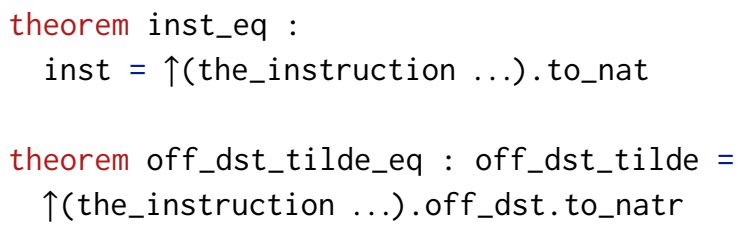

Here, we have omitted the arguments to the_instruction and the proofs of the theorems. The up arrow, which indicates the cast, can often be left implicit. The first theorem says that if we take the instruction computed from the constraints, encode it as a natural number less than $2^{63}$ in the natural way, and cast it to the finite field, the result is exactly inst. The second theorem says, similarly, that the AIR element off_dst_tilde is the field element corresponding to the off_dst bitvector component of the instruction. The function to_natr translates the given bitvector to a natural number; the " $r$ " at the end reflects the fact that the to_nat function in Lean's standard library takes 0 to be the most significant bit rather than the least significant bit, whereas to_natr does the opposite. We also prove uniqueness:

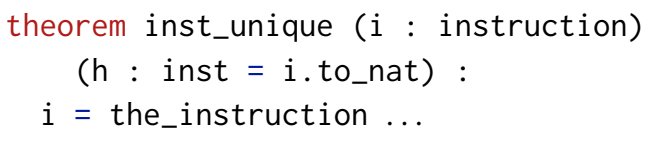

Interestingly, uniqueness is not required for the correctness theorem in Figure 1. The possibility that a field element might represent different instructions would make the nextstate relation nondeterministic, but it would not violate the theorem. But it is independently important to know that this nondeterminism does not arise, and we use that fact in subsequent work, when we want to prove that particular programs meet their specifications. For that purpose, we need to know that the original instruction can be recovered from its representation in the field.

\section{The Next State Relation}

The constraints that relate one state to the next are rather straightforward. The AIR has cells associated to each step $i \leq T$ of the execution trace. There are values $a p_{i}, f p_{i}$, and $p c_{i}$ representing the allocation pointer, frame pointer, and program counter at step $i$. There are also auxiliary values $d s t_{i}$ and res $_{i}$ for destination and result values associated with some of the instructions, as described by the CPU specification, and auxiliary values used in the constraints, as described by the Cairo whitepaper. Some of the auxiliary values are used to express the constraints as quadratic expressions, as required by the protocol.

For example, to say that the field value next_fp represents the correct value of the next frame pointer given the current register values, the state of memory, and the current instruction, the AIR uses the following constraint:

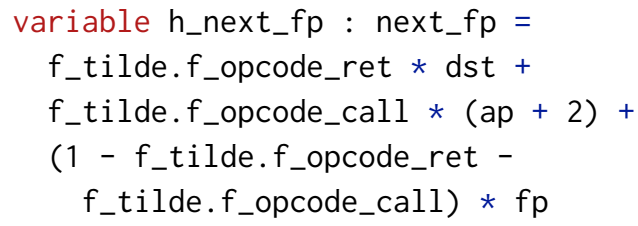

f_tilde.f_opcode_ret and f_tilde.f_opcode_call are the values of the corresponding flags, which were decoded by the instruction constraints. If the opcode_ret bit is 1 and the opcode_call bit is 0 , the constraint requires that the next frame pointer has the value dst; if opcode_ret is 0 and opcode_call is 1 , the constraint requires that the next frame pointer has the value $a p+2$; if both are 0 , the constraint requires that the frame pointer remains unchanged. Remember that if, for example, both flags are 1, the CPU semantics says that the next frame pointer can be anything at all; it is nondeterministic in that sense. This is not problematic, because the program itself is part of the specification shared by the prover and the verifier. The verifier can check that the instructions are indeed well-formed, and an end-toend verification that a Cairo program meets its specification will generally prove that fact along the way.

The claim that this constraint together with the constraints governing instructions, calculation of $d s t$, memory access, and so on all imply that the calculated next state agrees with the CPU semantic specification is stated simply as follows:

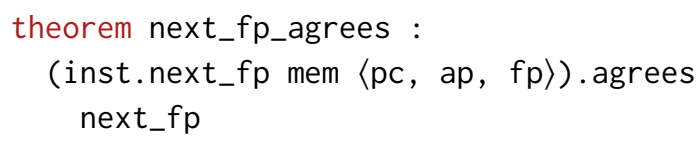

The parameters and hypotheses are left implicit, which is a good thing, because there are a lot of them, even for a simple fact like this one. Asking Lean to display the theorem statement shows that there are five hypotheses involving eight field values, the memory assignment, the instruction, and the associated tuple of flags. As one might expect, one of the most useful roles that the proof assistant played in our 
formalization was keeping track of the large array of data and assumptions, and making sure all the intermediate results were glued together correctly. The proof of this particular theorem involving little more than casing on the values of the flags, invoking previous results, and simplifying, with about eight lines of tactics.

\section{Permutations}

There are two types of constraints left to describe. Defining the next-step relation requires, in certain places, statements to the effect that a memory location $a$ contains a value $v$. The prover needs not only to encode this information in the AIR but also to convince the verifier that there is an assignment of values to the read-only memory that is consistent with these claims. The constraints that do this are described in Section 11. The correctness of the execution trace also requires knowing that address offsets stored in an instruction are integers in the interval $\left[0,2^{16}\right)$, and the Cairo compiler allows programmers to make similar assertions in their code. These range checks are handled by constraints described in Section 10. Both the memory constraints and range check constraints rely on an additional feature of the interactive proof protocol, the interaction step, that was alluded to in Section 4. In this section we explain how it works.

Suppose the prover has a sequence $a_{0}, a_{1}, \ldots, a_{n-1}$ of values in the field, uniformly spaced in the data, so that the prover can express uniform claims about these values using polynomial constraints. Remember that in general the verifier will not see this data; the prover will merely use the interactive proof system to convince the verifier of its existence.

Now suppose that to establish further claims about the data, the prover needs to list the elements in a different order. The prover adds a permutation $a_{0}^{\prime}, a_{1}^{\prime}, \ldots, a_{n-1}^{\prime}$, with additional polynomial constraints expressed in terms of those elements.

To establish correctness, the prover needs to convince the verifier that the second sequence is a permutation of the first. This is not easy to do. A STARK makes it possible to write down a constraint that relates, say, each pair $\left(a_{i}, a_{i}^{\prime}\right)$ to the next pair $\left(a_{i+1}, a_{i+1}^{\prime}\right)$, but the statement that the sequence $\left(a_{i}\right)_{i<n}$ is a reordering of $\left(a_{i}^{\prime}\right)_{i<n}$ cannot be expressed using local constraints of that form.

This is where the interaction step comes in. Let $p(x)$ be the polynomial given by $p(x)=\prod_{i<n}\left(x-a_{i}\right)$, and let $p^{\prime}(x)=$ $\prod_{i<n}\left(x-a_{i}^{\prime}\right)$. If the sequence $\left(a_{i}\right)_{i<n}$ is a permutation of $\left(a_{i}^{\prime}\right)_{i<n}$, then $p(x)-p^{\prime}(x)$ is the zero polynomial. If it isn't, then $p(x)-p^{\prime}(x)$ is a polynomial of degree at most $n$, and so has at most $n$ roots in the field. Suppose $n$ is much smaller than the size of the field, and let $\alpha$ be a randomly chosen element. Suppose also that the prover convinces the verifier that $p(\alpha)-p^{\prime}(\alpha)=0$. Then the verifier knows that either $\left(a_{i}\right)_{i<n}$ really is a permutation of $\left(a_{i}^{\prime}\right)_{i<n}$, or the prover was very lucky that the choice of $\alpha$ did not disprove that claim.

The interactive proof protocol uses a cryptographic hash instead of randomness. The STARK protocol enables the prover to commit to $a_{0}, a_{1}, \ldots, a_{n-1}$ and $a_{0}^{\prime}, a_{1}^{\prime}, \ldots, a_{n-1}^{\prime}$ and publish a certificate that these values meet the publicly shared constraints. Once it is published, a cryptographic hash $\alpha$ is generated. The prover then publishes a certificate that establishes that $p(\alpha)-p^{\prime}(\alpha)=0$.

To implement the argument in Lean, given any pair of finite tuples $a$ and $b$ indexed over a finite type $n$, we define the set of misleading choices of $\alpha$ as follows:

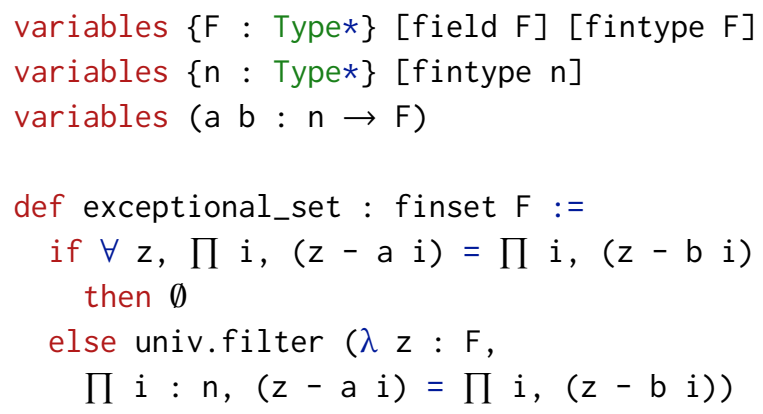

If the two polynomials are equal, the exceptional set is the empty set, which is to say, there are no misleading values. Otherwise, the exceptional set is the finite set of values that make the two sides equal. The facts we need to establish are, first, that the cardinality of the exceptional set is less than that of $n$, and, second, that unless the field element $z$ is in the exceptional set, equality of the two polynomials at $z$ implies that they are always equal.

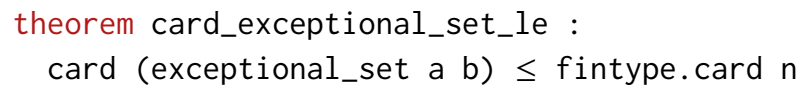

The first claim is proved by cases on the definition of the exceptional set. If it is the empty set, the claim is trivial, and otherwise the claim follows from the fact that the elements of the exceptional set are the roots of a nonzero polynomial of degree at most the cardinality of $n$. The second claim is immediate from the definition of the exceptional set, since the hypotheses imply that it cannot be equal to the second branch of the conditional.

In the proof of the final theorem in Figure 1, the existential quantifiers over bad 2 and bad 3 are witnessed by instances of exceptional_set. (We take bad1 to be the set of zeros of another polynomial, described in Section 11.) Note that the statement of the final theorem doesn't depend on the 
definition of exceptional_set or the details of how the bad sets are constructed. The theorem simply asserts the existence of finite sets that depend on the first 23 columns of data and not the final two, with the properties that (1) they are small, and (2) as long as the values of the interaction elements are outside those sets, the desired conclusion is guaranteed to hold.

\section{Range Checks}

We have seen that specifying that a trace cell represents a well-formed instruction requires in particular showing that certain other trace cells are field elements that are casts of integers in the interval $\left[0,2^{16}\right)$. Suppose $a_{0}, a_{1}, \ldots, a_{n-1}$ are the relevant values, let $\mathrm{rc}_{\min }$ be the minimum value, and let $\mathrm{rc}_{\max }$ be the maximum value. We can, without loss of generality, assume that every value between $\mathrm{rc}_{\min }$ and $\mathrm{rc}_{\max }$ occurs on the list, by padding the list with extra elements if necessary. We can convince the verifier that all the values are between $\mathrm{rc}_{\min }$ and $\mathrm{rc}_{\max }$ by dedicating another sequence of trace values $a_{0}^{\prime}, a_{1}^{\prime}, \ldots, a_{n-1}^{\prime}$, making $\mathrm{rc}_{\min }$ and $\mathrm{rc}_{\max }$ available to the verifier, and establishing the following claims:

- The sequence $\left(a_{i}^{\prime}\right)_{i<n}$ is a permutation of $\left(a_{i}\right)_{i<n}$.

- For each $i<n-1$, either $a_{i+1}=a_{i}$ or $a_{i+1}=a_{i}+1$.

- $a_{0}^{\prime}=\mathrm{rc}_{\min }$ and $a_{n-1}^{\prime}=\mathrm{rc}_{\max }$.

The first of these is handled as described in Section 9. We designate a sequence of trace elements $p_{0}, p_{1}, \ldots, p_{n-1}$ with the declaration $p:$ fin $(n+1) \rightarrow F$, and we add the following constraints:

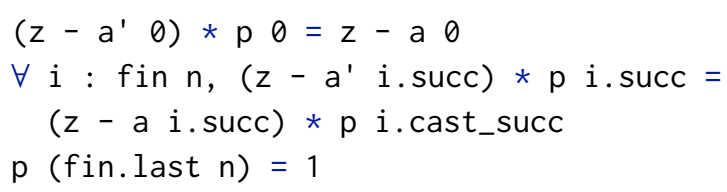

Here $i$. succ is Lean notation for $i+1$ as a value of type fin $(n+1)$, and i.cast_succ is notation for $i$ as a value of type fin $(n+1)$. These constraints guarantee that we have $\prod_{i<n}\left(z-a_{i}\right)=\prod_{i<n}\left(z-a_{i}^{\prime}\right)$, and if $z$ is generated by a hash, this equation offers the verifier a strong guarantee that the first condition is met. As described in Section 9, given

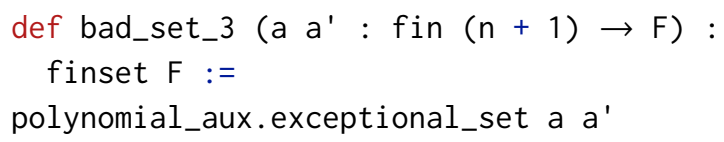

and the hypothesis $z \notin$ bad_set_3 a a', we have

lemma rc_permutation : $\forall i, \exists j, a i=a^{\prime} j$

For the second and third conditions, we simply add the following constraints:

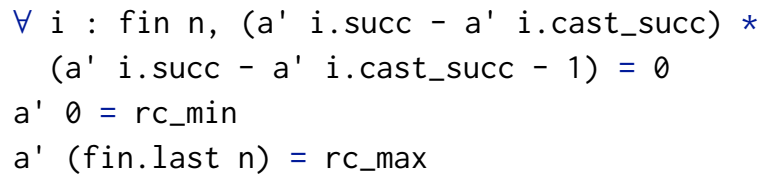

With the ultimate arrangement of data in the AIR, the values off_op0_tilde are included along the values a $j$ with an explicit embedding:

$$
\forall i, \text { a (embed_off_op } 0 \text { i) }=\text { off_opo_tilde } i
$$

With this, and the assumption rc_max $<2^{\wedge} 16$, we have

$$
\begin{aligned}
& \text { theorem off_op } \theta_{-} \text {in_range }(i: \text { fin } T): \\
& \exists \mathrm{k}: \mathbb{N}, k<2^{\wedge} 16 \wedge \text { off_op } \theta_{-} \text {tilde } i=\uparrow k
\end{aligned}
$$

We also have the analogous properties for off_op $\theta_{-} t i l d e$ and off_dst_tilde, which were assumed in Section 7.

\section{Memory}

During the course of its execution, a Cairo program will access memory locations $a_{0}, a_{1}, \ldots, a_{n-1}$. The prover has to establish the existence of a list of suitable values $v_{0}, v_{1}, \ldots, v_{n-1}$ for those memory locations. The constraints described in Section 8 guarantee that the values chosen by the prover are consistent with the semantics of the program execution. For example, if a program asserts the equality of the values in memory at locations $a_{i}$ and $a_{j}$, the constraints will guarantee that $v_{i}$ is equal to $v_{j}$.

But the prover also has to establish the values are consistent with each other, which is to say, there exists an assignment $\mathfrak{m}$ of values to the memory with the property that $\mathrm{m}\left(a_{i}\right)=v_{i}$ for every $i<n$. This is equivalent to saying that for every $i$ and $j$ less than $n$, if $a_{i}=a_{j}$, then $v_{i}=v_{j}$.

The constraints in the AIR that ensure this are similar to the range check constraints discussed in Section 10, with two additional twists. As in Section 10, we can assume that the set of values $\left\{a_{0}, a_{1}, \ldots, a_{n-1}\right\}$ is an interval by assigning 0 to unused memory locations between the smallest and largest values. If we designate trace elements $a_{0}^{\prime}, a_{1}^{\prime}, \ldots, a_{n-1}^{\prime}$ and $v_{0}^{\prime}, v_{1}^{\prime}, \ldots, v_{n-1}^{\prime}$, it suffices to establish the following:

- The sequence of pairs $\left(a_{i}^{\prime}, v_{i}^{\prime}\right)_{i<n}$ is a permutation of the sequence of pairs $\left(a_{i}, v_{i}\right)_{i<n}$.

- For each $i<n-1$, either $a_{i+1}^{\prime}=a_{i}^{\prime}$ or $a_{i+1}^{\prime}=a_{i}^{\prime}+1$.

- For each $i<n-1$, if $a_{i+1}^{\prime}=a_{i}^{\prime}$, then $v_{i+1}^{\prime}=v_{i}^{\prime}$.

The second and third of these are ensured by the following constraints:

$$
\begin{aligned}
& \forall i \text { : fin } n,\left(a^{\prime} i \text {.succ - } a^{\prime} i\right. \text {.cast_succ) * } \\
& \left(a^{\prime} \text { i.succ }-a^{\prime} \text { i.cast_succ }-1\right)=0 \\
& \forall i \text { : fin } n \text {, ( } v^{\prime} i \text {.succ - } v^{\prime} \text { i.cast_succ) * } \\
& \left(a^{\prime} \text { i.succ }-a^{\prime} \text { i.cast_succ }-1\right)=0
\end{aligned}
$$

Notice that the first of these constraints is needed to establish that the second one works as advertised.

The first twist is that to establish the first condition, it suffices to establish that the values $\left(a_{i}^{\prime}+\alpha v_{i}^{\prime}\right)_{i<n}$ are a permutation of the values $\left(a_{i}+\alpha v_{i}\right)_{i<n}$, provided that $\alpha$ has the property that $a_{i}^{\prime}+\alpha v_{i}^{\prime}$ is not equal to $a_{i}+\alpha v_{i}$ unless $a_{i}^{\prime}=a_{i}$ and $v_{i}^{\prime}=v_{i}$. But if the two quantities are equal and $v_{i}^{\prime} \neq v_{i}$, we have $\alpha=\left(a_{i}^{\prime}-a_{i}\right) /\left(v_{i}-v_{i}^{\prime}\right)$. Assuming $n$ is much smaller 
than the characteristic of $F$, there are a relatively small number of values $\alpha$ in the field with this property. So if we have the identity

$$
\prod_{i<n}\left(z-\left(a_{i}+\alpha v_{i}\right)\right)=\prod_{i<n}\left(z-\left(a_{i}^{\prime}+\alpha v_{i}^{\prime}\right)\right)
$$

for a randomly chosen $\alpha$ and a randomly chosen $z$, then first condition holds with high probability. This explains the expressions bad1 and bad 2 in the statement of the final correctness theorem: the first is the small set of possibly misleading $\alpha \mathrm{s}$, and the second is the small set of possibly misleading $z$ s. These are similar to the set bad3 defined for the range checks.

The second twist is that our global specification assumes that the prover and the verifier both have access to a partial specification $\mathrm{m}^{*}$ of values to memory locations. Remember, this is generally used to specify the Cairo program that has been executed, the agreed-upon inputs, and the claimed output. The prover has to convince the verifier that there is a memory function $\mathrm{m}$ that not only maps $a_{i}$ to $v_{i}$ for each $i<n$ but is also consistent with $\mathrm{m}^{*}$. One solution is to add the set of pairs $\left\{\left(a, \mathfrak{m}^{*}(a)\right)\right\}_{a \in \operatorname{dom}\left(\mathrm{m}^{*}\right)}$ to the list of pairs $\left(a_{0}, v_{0}\right),\left(a_{1}, v_{1}\right), \ldots,\left(a_{n-1}, v_{n-1}\right)$, but that is inefficient, because it requires adding an additional pair of constraints for each element of the domain of $\mathrm{m}^{*}$. Instead, we add $\left|\operatorname{dom}\left(\mathrm{m}^{*}\right)\right|$ pairs $(0,0)$ to the sequence $\ldots\left(a_{i}, v_{i}\right) \ldots$, and then we change the constraints to say that the sequence $\left(a_{i}^{\prime}, v_{i}^{\prime}\right)_{i<n}$ is a permutation of the sequence that results from replacing these pairs $(0,0)$ by the pairs $\left(a, \mathrm{~m}^{*}(a)\right)$. As explained by the whitepaper, it suffices to change the final constraint in Section 10 from $p$ (fin. last $n$ ) $=1$ to the following:

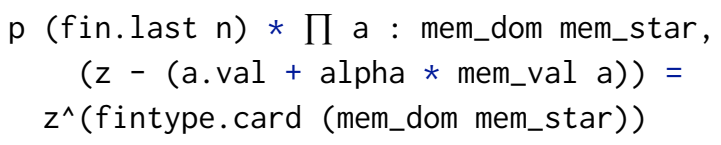

Assuming all the constraints are met, we define a function $\mathrm{m}$ from the sequences $\left(a_{i}^{\prime}\right)_{i<n}$ and $\left(v_{i}^{\prime}\right)_{i<n}$ :

$$
\begin{aligned}
& \text { def mem }\left(a^{\prime} v^{\prime}: \text { fin }(n+1) \rightarrow F\right): F \rightarrow F:= \\
& \lambda \text { addr, if } h: \exists i, a^{\prime} i=\operatorname{addr} \text { then } \\
& v^{\prime} \text { (classical. some } h \text { ) else } 0
\end{aligned}
$$

If a value addr occurs in the sequence $a^{\prime}$, we define mem add $r$ to be the corresponding value of $v^{\prime}$. We later show that the constraints imply that this value is unique. If addr does not occur in the sequence, we return 0 .

Given this definition of $m$ (i.e. mem in the formalization), we then show that the memory constraints imply the following:

- The memory assignment mem extends the partial assignment mem_star, which is ultimately specified in the inp parameter in the final correctness theorem in Figure 1.

- The pairs of values a i, $v$ i referred to in the onestep constraints satisfy mem $\left(\begin{array}{ll}a & i\end{array}\right)=\mathrm{v} i$.
We ultimately have to be explicit as to where the various memory accesses referred to in the cpu semantics are embedded in the list of pairs $\left(a_{i}, v_{i}\right)$. This information is specified in glue. lean, which relates the automatically generated polynomial constraints to the informal ones in the whitepaper description.

This concludes our description of the constraints and our presentation of the main theorem.

\section{Conclusions}

We have verified the correctness of an algebraic encoding of the Cairo CPU semantics. The encoding is used to publish, on blockchain, efficient proofs of the correctness of claims about machine-code execution with respect to that semantics. The verification is valuable in its own right, but it is also an important stepping-stone toward verification of higher-level programs in the Cairo programming language with respect to higher-level descriptions of their behavior.

A notable feature of our work is that the encoding that we have verified is already in commercial use. It is used by the StarkEx platform to carry out cryptocurrency exchanges efficiently, and it is used by StarkNet, which enables developers to implement similarly efficient computational transactions on blockchain. This places a high premium on establishing the correctness of the encoding. The whitepaper proof is quite technical, since it requires reasoning about a number of algebraic constraints and fitting a number of small results together in just the right way. It is therefore reassuring that the verification of the whitepaper went through straightforwardly and confirmed the correctness of the implementation.

\section{Related Work}

The formalization we report on here only addresses the relationship between the machine semantics and its algebraic representation. It is therefore similar to projects that model processor instruction sets, such as the $\mathrm{x} 86$ instruction set $[15,18]$ and the Ethereum virtual machine $[5,21,22]$. In comparison to x86 and even EVM, however, the Cairo machine model is fairly simple. We have described some of the novel features of the model, such as the fact that it has a read-only memory and operates on values from a finite field.

We do not know of any project that verifies an algebraic encoding of execution traces. Another approach to verifying computation using cryptographic protocols involves compiling each individual program to a set of polynomial constraints that describe its execution, unrolling loops and bounding the number of iterations, and so on. This method suffers from some well-known drawbacks, discussed in [19, Section 1.1]. Fournet et al. [17] verify the correctness of such transformations, as carried out by Pinocchio [14, 27].

The more general goal of verifying smart contracts in various senses has become too big an industry to survey here [31]. Jiao et al. [23] and Ribeiro et al. [28] verify programs 
written in a subset of Solidity with respect to a high-level description of the semantics, and Bhargavan et al. [11] verify smart contracts written in Solidity by translating them to $F^{\star}$. Annenkov et al. [6] provide means of defining and verifying smart contracts in Coq and then extracting code for various blockchain platforms. A number of systems provide means of verifying the correctness of cryptographic protocols and their implementations, including [1-4, 9, 12, 29].

Our long-term goal of verifying Cairo programs with respect to a machine semantics (and hence, with the results here, with respect to the final STARK certificates) makes it similar to other projects that are designed to verify software with respect to a machine level semantics, such as CompCert [25], CakeML [24], and VST [13]. Once again, the specifics of the Cairo platform and its applications give rise to novel aspects of the verification task that we will continue to report on in the future.

\section{References}

[1] Carmine Abate, Philipp G. Haselwarter, Exequiel Rivas, Antoine Van Muylder, Théo Winterhalter, Catalin Hritcu, Kenji Maillard, and Bas Spitters. 2021. SSProve: A Foundational Framework for Modular Cryptographic Proofs in Coq. (2021), 1-15. https://doi.org/10.1109/ CSF51468.2021.00048

[2] José Bacelar Almeida, Endre Bangerter, Manuel Barbosa, Stephan Krenn, Ahmad-Reza Sadeghi, and Thomas Schneider. 2010. A Certifying Compiler for Zero-Knowledge Proofs of Knowledge Based on Sigma-Protocols. In European Symposium on Research in Computer Security (ESORICS) 2010, Dimitris Gritzalis, Bart Preneel, and Marianthi Theoharidou (Eds.). Springer, 151-167. https://doi.org/10.1007/978-3642-15497-3_10

[3] José Bacelar Almeida, Manuel Barbosa, Endre Bangerter, Gilles Barthe, Stephan Krenn, and Santiago Zanella Béguelin. 2012. Full proof cryptography: verifiable compilation of efficient zero-knowledge protocols. In Conference on Computer and Communications Security (CCS) 2012, Ting Yu, George Danezis, and Virgil D. Gligor (Eds.). ACM, 488-500. https://doi.org/10.1145/2382196.2382249

[4] José Bacelar Almeida, Manuel Barbosa, Manuel L. Correia, Karim Eldefrawy, Stéphane Graham-Lengrand, Hugo Pacheco, and Vitor Pereira. 2021. Machine-checked ZKP for NP relations: Formally Verified Security Proofs and Implementations of MPC-in-the-Head. In Computer and Communications Security (CCS) 2021, Yongdae Kim, Jong Kim, Giovanni Vigna, and Elaine Shi (Eds.). ACM, 2587-2600. https://doi.org/10.1145/3460120.3484771

[5] Sidney Amani, Myriam Bégel, Maksym Bortin, and Mark Staples. 2018. Towards verifying ethereum smart contract bytecode in Isabelle/HOL. In Certified Programs and Proofs (CPP) 2018, June Andronick and Amy P. Felty (Eds.). ACM, 66-77. https://doi.org/10.1145/3167084

[6] Danil Annenkov, Mikkel Milo, Jakob Botsch Nielsen, and Bas Spitters. 2021. Extracting smart contracts tested and verified in Coq. In Certified Programs and Proofs (CPP) 2021, Catalin Hritcu and Andrei Popescu (Eds.). ACM, 105-121. https://doi.org/10.1145/3437992.3439934

[7] László Babai. 1985. Trading Group Theory for Randomness. In ACM Symposium on Theory of Computing (SToC) 1985, Robert Sedgewick (Ed.). ACM, 421-429. https://doi.org/10.1145/22145.22192

[8] László Babai, Lance Fortnow, and Carsten Lund. 1991. NonDeterministic Exponential Time has Two-Prover Interactive Protocols. Comput. Complex. 1 (1991), 3-40. https://doi.org/10.1007/BF01200056

[9] Gilles Barthe, Daniel Hedin, Santiago Zanella Béguelin, Benjamin Grégoire, and Sylvain Heraud. 2010. A Machine-Checked Formalization of
Sigma-Protocols. In Computer Security Foundations Symposium (CSF) 2010. IEEE Computer Society, 246-260. https://doi.org/10.1109/CSF. 2010.24

[10] Eli Ben-Sasson, Iddo Bentov, Yinon Horesh, and Michael Riabzev. 2018. Scalable, transparent, and post-quantum secure computational integrity. IACR Cryptol. ePrint Arch. 2018 (2018), 46. http: //eprint.iacr.org/2018/046

[11] Karthikeyan Bhargavan, Antoine Delignat-Lavaud, Cédric Fournet, Anitha Gollamudi, Georges Gonthier, Nadim Kobeissi, Natalia Kulatova, Aseem Rastogi, Thomas Sibut-Pinote, Nikhil Swamy, and Santiago Zanella Béguelin. 2016. Formal Verification of Smart Contracts: Short Paper. In Programming Languages and Analysis for Security (PLAS) 2016, Toby C. Murray and Deian Stefan (Eds.). ACM, 91-96. https://doi.org/10.1145/2993600.2993611

[12] David Butler, Andreas Lochbihler, David Aspinall, and Adrià Gascón. 2021. Formalising $\Sigma$-Protocols and Commitment Schemes Using CryptHOL. J. Autom. Reason. 65, 4 (2021), 521-567. https://doi.org/10. 1007/s10817-020-09581-w

[13] Qinxiang Cao, Lennart Beringer, Samuel Gruetter, Josiah Dodds, and Andrew W. Appel. 2018. VST-Floyd: A Separation Logic Tool to Verify Correctness of C Programs. J. Autom. Reason. 61, 1-4 (2018), 367-422. https://doi.org/10.1007/s10817-018-9457-5

[14] Craig Costello, Cédric Fournet, Jon Howell, Markulf Kohlweiss, Benjamin Kreuter, Michael Naehrig, Bryan Parno, and Samee Zahur. 2015. Geppetto: Versatile Verifiable Computation. In Symposium on Security and Privacy (SP) 2015. IEEE Computer Society, 253-270. https://doi.org/10.1109/SP.2015.23

[15] Sandeep Dasgupta, Daejun Park, Theodoros Kasampalis, Vikram S. Adve, and Grigore Rosu. 2019. A complete formal semantics of x8664 user-level instruction set architecture. In Programming Language Design and Implementation (PLDI) 2019, Kathryn S. McKinley and Kathleen Fisher (Eds.). ACM, 1133-1148. https://doi.org/10.1145/ 3314221.3314601

[16] Leonardo Mendonça de Moura, Soonho Kong, Jeremy Avigad, Floris van Doorn, and Jakob von Raumer. 2015. The Lean Theorem Prover (System Description). In Conference on Automated Deduction (CADE) 2015, Amy P. Felty and Aart Middeldorp (Eds.). Springer, Berlin, 378388. https://doi.org/10.1007/978-3-319-21401-6_26

[17] Cédric Fournet, Chantal Keller, and Vincent Laporte. 2016. A Certified Compiler for Verifiable Computing. In Computer Security Foundations Symposium (CSF) 2016. IEEE Computer Society, 268-280. https://doi. org/10.1109/CSF.2016.26

[18] Shilpi Goel, Anna Slobodová, Rob Sumners, and Sol Swords. 2020. Verifying $\mathrm{x} 86$ instruction implementations. In Certified Programs and Proofs (CPP) 2020, Jasmin Blanchette and Catalin Hritcu (Eds.). ACM, 47-60. https://doi.org/10.1145/3372885.3373811

[19] Lior Goldberg, Shahar Papini, and Michael Riabzev. 2021. Cairo - a Turing-complete STARK-friendly CPU architecture. Cryptology ePrint Archive, Report 2021/1063. https://ia.cr/2021/1063.

[20] Shafi Goldwasser, Silvio Micali, and Charles Rackoff. 1989. The Knowledge Complexity of Interactive Proof Systems. SIAM F. Comput. 18, 1 (1989), 186-208. https://doi.org/10.1137/0218012

[21] Everett Hildenbrandt, Manasvi Saxena, Nishant Rodrigues, Xiaoran Zhu, Philip Daian, Dwight Guth, Brandon M. Moore, Daejun Park, Yi Zhang, Andrei Stefanescu, and Grigore Rosu. 2018. KEVM: A Complete Formal Semantics of the Ethereum Virtual Machine. In Computer Security Foundations Symposium (CSF) 2018. IEEE Computer Society, 204-217. https://doi.org/10.1109/CSF.2018.00022

[22] Yoichi Hirai. 2017. Defining the Ethereum Virtual Machine for Interactive Theorem Provers. In Financial Cryptography and Data Security (FC) 2017, Michael Brenner, Kurt Rohloff, Joseph Bonneau, Andrew Miller, Peter Y. A. Ryan, Vanessa Teague, Andrea Bracciali, Massimiliano Sala, Federico Pintore, and Markus Jakobsson (Eds.). Springer, 520-535. https://doi.org/10.1007/978-3-319-70278-0_33 
[23] Jiao Jiao, Shuanglong Kan, Shang-Wei Lin, David Sanán, Yang Liu, and Jun Sun. 2020. Semantic Understanding of Smart Contracts: Executable Operational Semantics of Solidity. In Security and Privacy (SP) 2020. IEEE, 1695-1712. https://doi.org/10.1109/SP40000.2020.00066

[24] Ramana Kumar, Magnus O. Myreen, Michael Norrish, and Scott Owens. 2014. CakeML: a verified implementation of ML. In Principles of Programming Languages (POPL) 2014, Suresh Jagannathan and Peter Sewell (Eds.). ACM, 179-192. https://doi.org/10.1145/2535838.2535841

[25] Xavier Leroy. 2009. Formal verification of a realistic compiler. Commun. ACM 52, 7 (2009), 107-115. https://doi.org/10.1145/1538788.1538814

[26] The mathlib community. 2020. The lean mathematical library. In Certified Programs and Proofs (CPP) 2020, Jasmin Blanchette and Catalin Hritcu (Eds.). ACM, 367-381. https://doi.org/10.1145/3372885.3373824

[27] Bryan Parno, Jon Howell, Craig Gentry, and Mariana Raykova. 2016 Pinocchio: nearly practical verifiable computation. Commun. ACM 59, 2 (2016), 103-112. https://doi.org/10.1145/2856449
[28] Maria Ribeiro, Pedro Adão, and Paulo Mateus. 2020. Formal Verification of Ethereum Smart Contracts Using Isabelle/HOL. In Logic, Language, and Security - Essays Dedicated to Andre Scedrov on the Occasion of His 65th Birthday, Vivek Nigam, Tajana Ban Kirigin, Carolyn L. Talcott, Joshua D. Guttman, Stepan L. Kuznetsov, Boon Thau Loo, and Mitsuhiro Okada (Eds.). Springer, 71-97. https://doi.org/10.1007/9783-030-62077-6_7

[29] Nikolaj Sidorenco, Sabine Oechsner, and Bas Spitters. 2021. Formal security analysis of MPC-in-the-head zero-knowledge protocols. In Computer Security Foundations Symposium (CSF) 2021. IEEE, 1-14. https://doi.org/10.1109/CSF51468.2021.00050

[30] Nick Szabo. 1997. Formalizing and Securing Relationships on Public Networks. First Monday 2, 9 (1997).

[31] Palina Tolmach, Yi Li, Shang-Wei Lin, Yang Liu, and Zengxiang Li. 2021. A Survey of Smart Contract Formal Specification and Verification. ACM Comput. Surv. 54, 7 (2021). https://doi.org/10.1145/3464421 